\title{
ANTICOAGULATION THERAPY
}

\section{Results of RE-ALIGN disappoint}

The oral direct thrombin inhibitor dabigatran should not be used in patients with mechanical heart valves, owing to an increased risk of thromboembolic events and bleeding. This recommendation follows the publication of findings from RE-ALIGN, a phase II, randomized, open-label trial in which dabigatran was compared with warfarin in patients with a mechanical aortic or mitral valve prosthesis. The trial was terminated early, in December 2012, and warnings about the use of dabigatran in this patient population were subsequently issued by the European Medicines Agency and the FDA. Data from the study were presented at the 2013 ESC Congress by Frans Van de Werf, who explained that "dabigatran etexilate has been shown to be effective and safe in other indications", but that the mechanisms of valve thrombosis, which are different to those associated with atrial fibrillation, could partially explain failure of dabigatran in this setting.

RE-ALIGN was conducted over a 12-week period at 39 centres across 10 countries. Participants were due to undergo implantation of a mechanical bileaflet valve (aortic, mitral, or both; population A), or had received a mechanical mitral valve (or both mitral and aortic valves) $>3$ months

Original article Eikelboom, J. W. et al. Dabigatran versus warfarin in patients with mechanical heart valves. N. Engl. J. Med. doi:10.1056/NEJMoa1300615 before enrollment in the trial (population B). Patients were randomly assigned to receive anticoagulation therapy with dabigatran $(n=168)$ or warfarin $(n=84)$. Dabigatran dose was 150-300 mg twice daily, depending on renal function, with a target trough plasma level of $\geq 50 \mathrm{ng} / \mathrm{ml}$. Warfarin dose was tailored to achieve an international normalized ratio of 2-3 for patients at low risk of thromboembolic events, and 2.5-3.5 for those at high risk. At the end of the initial study period, $59 \%$ of patients in the dabigatran group and $70 \%$ of those in the warfarin group decided to enter the extension phase (RE-ALIGN-EX), which had a planned duration of up to 84 months. Stroke and myocardial infarction occurred in $5 \%$ and $2 \%$, respectively, of patients receiving dabigatran (all in population $\mathrm{A}$ ), whereas none of the individuals in the warfarin group experienced these events. In addition, valve thrombosis without symptoms was detected in $3 \%$ of patients receiving dabigatran, but not in the warfarin group. The incidence of bleeding events was increased by more than twofold with dabigatran compared with warfarin (27\% versus $12 \%$; HR 2.45, 95\% CI 1.23-4.86, $P=0.01)$. All cases of major bleeding occurred in population A within 1 week of surgery.
In their report, the RE-ALIGN investigators propose several reasons for the poor outcomes associated with dabigatran, including inadequate plasma levels of the drug, and mechanisms of action. "In patients with a mechanical heart valve, coagulation activation and thrombin generation seem to be partially induced by exposure of blood to the artificial surfaces of the valve leaflets and sewing ring (contact thrombosis)," explained Professor Van de Werf in a press conference. "Warfarin is more effective in the setting of mechanical valves because it inhibits synthesis of factor IX ... [whereas] dabigatran exclusively inhibits thrombin.” In an Editorial that accompanied the trial report, Elaine Hylek concludes that, although the results of RE-ALIGN are disappointing, the trial was subject to several limitations and "there is a palpable downside to potential premature abandonment of research into the use of such drugs in patients with mechanical heart valves".

\section{Alexandra Roberts}

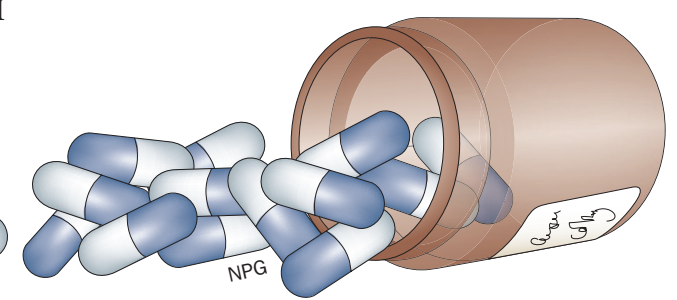

